\title{
È possibile coniugare risparmio e miglioramento nelle aziende usl?
}

Sergio Sabbatanio

\begin{abstract}
The present study aimed to evaluate if it's possible to reduce the costs for the anti-infective medicine in a ward for the infectious disease. Analytic accounts data, related to the cost for three kind of drugs (antibiotic, antimicrobic and antriretroviral) will be presented. The analytic account is referred to the Bologna USL Firm (Presidi ospedalieri Bellaria and Maggiore) and to the Hospital Unit for the infectious disease (Ospedale Maggiore) in the year 1999. The Hospital Unit for the infectious disease consists of two ward for day of stay ( 32 beds), one day hospital for 10 beds and one divisional outpatients' clinic. In 1999 there were 650 hospitalized (and discharged) patients and 783 day hospital patients.

The overall cost for the Bologna USL Firm was L. 11.068.023.000, accounting L. 7.253.846.000 (65.5\%) for the antiretroviral drug, L. 1.958.115.000 (17\%) for the antibiotic drug and L. 1.856.062.000 (16.8\%) for the antimicrobic drug and other antinfective medicine.

The expenditure for the Hospital Unit for the infectious disease was L. 7.543.851.000, accounting L.

7.008.163.000 (92.9\%) for the antiretroviral drug, L. 256.167 .000 (3.4\%) for the antibiotic drug and L. 279.521.000 $(3.7 \%)$ for the antimicrobic drug and other antinfective medicine.
\end{abstract}

Farmeconomia e percorsi terapeutici 2000; 1 (2): $97-101$

\section{INTRODUZIONE}

Con il processo di aziendalizzazione in atto nella sanità italiana, la domanda "quanto è costato" è diventata la questione più insidiosa che i dirigenti, deputati al processo di pianificazione strategica aziendale (c.d. decisori), pongono ai clinici, ai medici e ai biologi che si occupano dei pazienti nei laboratori.

Nella nuova gestione della sanità uno dei temi sul quale maggiormente ci si sta concentrando è l'analisi dei costi, perché questi sono al centro di molte scelte strategiche a livello aziendale.

Dopo la Legge 833 (istitutiva nel 1979 del S.S.N.) il processo di trasformazione che ha "sconvolto" la sanità pubblica tramite i decreti legislativi 502/192 e 517/93, sul riordino del S.S.N., ha determinato l'aziendalizzazione delle U.S.L. attribuendo loro autonomia organizzativa, patrimoniale, contabile, gestionale e tecnicaii.

Per quanto riguarda il ruolo dei sanitari tutto ciò ha determinato la responsabilizzazione dei medici sui risultati conseguiti, subordinando la conferma nella dirigenza alla verifica dei traguardi ottenutiiii. Con l'introduzione di indicatori di efficienza e l'attivazione di strumenti per la promozione della qualità dei servizi si sono individuate le modalità per controllare $\mathrm{i}$ risultati. La prospettiva strategica è indirizzata a stimolare e a monitorare i processi di autoriz- zazione e di accreditamento, elemento determinante per equiparare la sanità italiana a quella europea.

Grazie al Decreto ministeriale del 14 dicembre 1994, che definisce le tariffe delle prestazioni di assistenza ospedaliera (DRGS) e al Decreto ministeriale del giugno 1997, che le ha aggiornate, si sono potuti stabilire i costi degli atti sanitari. In altri termini è stato definito quanto il committente (l'Azienda USL) deve pagare al presidio ospedaliero per i singoli ricoveri effettuati nelle diverse Unità operative ospedaliere. Con il Decreto ministeriale del 22 luglio 1996 si sono inoltre definite le prestazioni di assistenza ospedaliera ambulatoriale erogabili nell' ambito del S.S.N., le relative tariffe massime e i codici.

Il Decreto legislativo n. 124 del 29.4.1998 (c.d. "Sanitometro") ha reinquadrato il sistema di partecipazione al costo delle prestazioni sanitarie e al regime delle esenzioni. Infine il Decreto Legge 229/99 "Riforma ter" ha stabilito l'attuazione della delega contenuta nella Legge 419/98 per la "Razionalizzazione" del S.S.N.. Obbiettivo dichiarato di quest'ultima riforma è Razionalizzare l'impianto organizzativo del Sistema, spingendolo verso una maggiore efficienza, qualità ed equità. In questa nuova visione le USL rimangono organismi pubblici, ma sono organizzate come aziende private con criteri imprenditoriali (1). a Unità Operativa di Malattie Infettive Osp. Maggiore Bologna

\section{Nota i}

Nella "nuova sanità" $i$ medici e i biologi impiegati nelle Az. USL vengono chiamati professional.
Nota ii
Alla fine degli anni Ottanta si è diffuso in
Italia un nuovo paradigma aziendale denominato "New Public Management" (N.P.M.), ove tra l'altro venivano introdotte logiche di competitività tra le diverse organiz- zazioni pubbliche, messe in concorrenza tra loro e con le imprese private, attraverso meccanismi finanziari d'incentivazionel penalizzazione (1). 


\section{Nota iii}

Con la N.P.M. viene garantita alla dirigenza tecnico-amministrativa pubblica la possibilità di gestire l'organizzazione secondo "criteri di discrezionalità professionale e manageriale" riducendo progressivamente $i$ limiti procedurali e amministrativi finora esistenti (1).

\section{Nota iv}

Sono esclusi da questo tipo di finanziamento, in quanto non ̀̀ possibile quantificare la singola prestazione: la prevenzione, le malattie rare, $i$ trapianti d'organo, l'assistenza ai cronici, servizi d'emergenza, $i$ programmi sperimentali d'assistenza, gli accertamenti preventivi sui donatori.

\section{Nota $\mathbf{v}$}

Le unità operative non sono più viste nell'ambito aziendale come semplice "braccio operativo", bensì, vengono ridefinite come Unità dotate di autonomia, responsabilizzate sulle risorse consumate e sui risultati conseguiti.

\section{Nota vi \\ Così si spiegano i costi con il variare dei volumi di produzione. I costi fissi sono quelli che non subiscono modifiche in relazione alle quantità prodotte (es. gli affitti, le polizze assicurative, gli appalti per la manutenzione delle attrezzature e in parte $i$ costi del personale). I costi variabili cambiano in modo proporzionale al volume della produzio- ne, cioè in base al livello di attività.}

Come si può desumere da queste brevi note introduttive la gestione sanitaria si è caratterizza, negli anni Novanta, verso un'impostazione economicistica. Le USL e le Aziende ospedaliere si sono dotate di un sistema di contabilità economico-patrimoniale organizzato per centri di costo. In questo contesto la minimizzazione dei costi (regola generale di razionalità economica) diventa nelle aziende sanitarie, remunerate prevalentemente a budget o a prestazione ${ }^{\mathrm{iv}}$, elemento qualificante sia della gestione complessiva sia delle singole Unità operativev ${ }^{v}$.

I presupposti metodologici di questa gestione sono:

- centralità del ruolo sanitario nel processo di controllo economico della propria Unità;

- attivazione di un controllo interno e non esterno all'Unità Operativa;

- assunzione a riferimento dell'Unità organizzativa come Unità di analisi.

Va precisato che, nelle intenzioni dei Legislatori, l'avere posto l'accento sui costi non dovrebbe fare assurgere il risparmio (in sé) al centro degli elementi valutativi; questo aspetto dovrebbe arricchire invece la competenza professionale di un'ulteriore valenza, stimolando i medici a conoscere gli aspetti economici, per migliorare le modalità con cui si utilizzano le risorse. In altri termini il professional "ideale" dovrebbe essere un accorto gestore delle risorse senza perdere l'identità di medico.

Nell'analisi economica dei processi produttivi aziendali i costi si possono dividere in fissi e variabili vi. Di solito un'Unità Operativa ospedaliera è caratterizzata da una preponderanza di costi fissi (il personale) e da costi variabili sostanzialmente contenuti vii (beni di consumo - farmaci). Occorre fare questa precisazione perché è evidente che una struttura dei costi caratterizzata da una forte prevalenza di costi fissi appare rigida e scarsamente influenzabile da interventi di "Razionalizzazione". In questo caso i margini di manovra di un processo di budget appaiono scarsi e poco comprimibili; si può influire, potenzialmente, sulle dotazioni organiche mediche (in genere solo mediante pensionamenti e trasferimenti), mentre la manovra può essere più incisiva sul personale infermieristico, più facilmente ricollocabile.

In questo ambito si può operare positivamente sui ricavi grazie a un incremento di efficienza $^{\text {viii }}$ a cui, teoricamente, dovrebbe corrispondere un aumento dei volumi produttivi con una stabilità dei costi fissi.

Precisiamo che l'Azienda USL, da un punto di vista dei modelli aziendali, è considerata un'organizzazione multiprodotto, poichè si rilevano molti fattori produttivi, ma solo alcuni sono impiegati esclusivamente nella produzio- ne di un servizio. La maggior parte delle risorse contribuisce alla realizzazione di più prestazioni. Quando si osserva un rapporto immediato causa effetto tra prodotto e costo si realizza un costo diretto (beni di consumo, farmaci, personale direttamente collegato al prodotto). Altrimenti si definiscono costi indiretti quelli che non sono automaticamente imputabili all'oggetto di riferimento; in questo caso i costi vengono ripartiti tra le diverse U.O. e i Servizi in funzione di una grandezza nota, assunta come base per effettuare i "ribaltamenti" ixe con questa modalità si ripartiscono le spese per i costi degli affitti, i costi somministrativi i servizi di manutenzione.

I sistemi di contabilità analitica puntano alla rilevazione dei costi consuntivi medi di prodotto (costi medi per giornata di degenza o per ricovero o per prestazione ambulatoriale erogata). Per descrivere la complessità del processo sarebbe necessario un maggiore spazio. Per una più completa trattazione si rimanda a testi specifici di economia sanitaria $(2,3,4,5,6,7)$.

I farmaci antinfettivi sono la classe più frequentemente utilizzata nelle U.O. di malattie infettive. Tra i prodotti antinfettivi gli antiretrovirali incidono più significativamente nel budget di questi reparti.

Le U.O. di malattie infettive (in particolare quelle che per motivi di collocazione geografica si sono trovate storicamente in prima linea già dalla metà degli anni Ottanta nel contrastare l'epidemia di AIDS) hanno tradizionalmente acquisito un forte credito nei confronti dei pazienti portatori d'infezione HIV. Questa tendenza ha determinato una discreta concentrazione dei pazienti in un numero relativamente limitato di centri clinici, con la migrazione di una parte di malati da alcune regioni verso reparti ritenuti (a torto o a ragione) più specializzati. Come è noto l'avvento dell'HAART ha consentito di ottenere un notevole miglioramento della prognosi quod vitam dei pazienti HIV positivi. Ciò ha portato a una riduzione sensibile del numero di ricoveri e delle giornate di degenza dei pazienti con $\operatorname{AIDS}^{\mathrm{x}}$ nei reparti di malattie infettive.

Contemporaneamente si è osservata l'espansione massiva della spesa sanitaria per la cura ambulatoriale di questi pazienti con farmaci antiretrovitali.

Scopo di questo studio è stimare, alla luce dell'analisi dei costi per la cura con farmaci antinfettivi in un reparto di malattie infettive, quale può essere lo spazio di manovra per ridurre la spesa farmaceutica, rimanendo in un ambito di appropriatezza e di massima qualità dell'intervento sanitario. Verranno per questa finalità presentati i dati di contabilità analitica, relativi alla spesa per le tre categorie di farmaci (antibiotici, antimicotici, antiretrovirali) del- 
l'Azienda USL e dell'U.O. malattie infettive e si porranno in discussione alcune considerazioni per ridurre la spesa, nella prospettiva di migliorarne la qualità.

\section{MATERIALI E METODI}

Si è compiuta un' analisi contabile dei costi sostenuti nel 1999 dall' Azienda USL città di Bologna (Presidi Ospedalieri Maggiore e Bellaria) e dall'U.O. di malattie infettive in relazione al consumo di farmaci antinfettivi. La spesa totale è stata suddivisa analizzando quella sostenuta per antiretrovirali, antibiotici, antimicotici e altri farmaci antinfettivi. Occorre precisare che l'U.O.M.I. è strutturata in due reparti di degenza con 32 posti letto, un Day Hospital che conta 10 posti letto e un ambulatorio divisionale.

Nel 1999 i pazienti ricoverati (e dimessi) nel reparto di degenza sono stati 650 e 783 i ricoverati presso il D.H.; di questi 475 sono residenti fuori Bologna.

\section{RISULTATI}

Considerando che,nell' ambito dei due Presidi Ospedalieri Bellaria e Maggiore dell' Azienda USL città di Bologna, la spesa totale per farmaci, in prontuario ed extraprontuario terapeutico, è stata nel 1999 pari a £. 18.914.955.000, la spesa complessiva per la classe dei farmaci antinfettivi è stata di £. 11.068.023.000 (58,5\%). La Tabella 1 consente di visualizzare la spesa sostenuta nei due presidi ospedalieri per farmaci antibiotici, antimicotici ed altri (antiprotozoari, antivirali e antielmintici) e per la terapia antiretrovirale.

La spesa per farmaci antinfettivi sostenuta nel 1999 dall'U.O. di Malattie Infettive dell'Ospedale Maggiore è invece presentata nella Tabella 2.

Come si può osservare, la spesa per farmaci antiretrovirali sostenuta dall'U.O.M.I. per la cura dell'infezione HIV richiede quasi il $93 \%$ della spesa complessiva.

A carico dell'U.O.M.I. è il 96,6\% della spesa per farmaci antiretrovirali erogata dall'intera Az. USL, mentre per gli antibiotici l'onere è pari al $13,08 \%$, e per il terzo raggruppamento (antimicotici, anti elimintici ecc.) è pari al 15,06\%

\section{DISCUSSIONE}

L'analisi contabile dei costi sostenuti nel 1999 nell'ambito dell' Azienda Città di Bologna per farmaci antinfettivi dimostra un forte impegno per la cura dei pazienti HIV positivi mediante la TARV. Questi pazienti, come è noto, vengono trattati prevalentemente presso l'ambulatorio e il Day Hospital del reparto di malattie infettive (centro di costo specifico). L'esperienza maturata in questi anni dimostra che difficilmente questa spesa nel prossimo futuro potrà essere sensibilmente ridotta in quanto i protocolli adottati prevedono l'utilizzo di tre o più farmaci $^{\mathrm{xi}}$

È auspicabile che una modesta riduzione di spesa (per i casi trattati con tre farmaci) si possa realizzare nel corso dell'anno 2000: infatti una porzione considerevole di pazienti curati con cocktail terapeutici che prevedevano inibitori delle Proteasi (I.P.) sono passati all'utilizzo della nuova classe di farmaci inibitori non nucleosidici della Transcriptasi Inversa (N.N.R.T.) $^{\text {xii }}$.

Come è noto questi farmaci sono entrati nell'uso corrente nel 1999 (Nevirapina) e nel primo semestre 2000 (Efavirenz), dopo essere stati utilizzati con successo in protocolli sperimentali $(9,10,11,12)$ e per uso compassionevole, . Questi due prodotti sono relativamente meno costosi degli I.P. che comunque rimangono punti di riferimento importanti della terapia antiretrovirale. Sono in corso studi caso controllo, in pazienti che sono passati da cocktails con I.P. ad associazioni di F. con N.N.R.T., per

\begin{tabular}{||l|c|c|}
\hline Antiretrovirali & L. 7.253.846.000 & $65.5 \%$ \\
\hline \hline Antibiotici & L. 1.958 .115 .000 & $17.7 \%$ \\
\hline \hline Antimicotici e altri antinfettivi & L. 1.856 .062 .000 & $16.8 \%$ \\
\hline Totale & L. 11.068.023.000 & $\mathbf{1 0 0 \%}$ \\
\hline
\end{tabular}

\begin{tabular}{||l|c|c|}
\hline Antiretrovirali & L. 7.008 .163 .000 & $92.9 \%$ \\
\hline \hline Antibiotici & L. 256.167 .000 & $3.4 \%$ \\
\hline \hline Antimicotici e altri antinfettivi & L. 279.521 .000 & $3.7 \%$ \\
\hline Totale & L. $\mathbf{7 . 5 4 3 . 8 5 1 . 0 0 0}$ & $\mathbf{1 0 0 \%}$ \\
\hline
\end{tabular}

\section{Tabella 1}

Spesa per farmaci antinfettivi (1999) Presidi Ospedalieri Bellaria e Maggiore (Az. USL città di Bologna).

\section{Tabella 2}

Spesa per farmaci antinfettivi (1999) U.O. malattie infettive Ospedale Maggiore (Az. USL città di Bologna).

U.O. di malattie

infettive che seguono,

numero importan pazienti HIV positivi.

Più oltre si motiverà

Nota viii

Non si può però prescindere da una

dell'appropriatezza dell'intervento e inoltre incremento dei cost

\section{ta ix}

sibile una requentemente) si aziendale tendente ad evitare calcoli laboriosi i dilevazione costosi per spese di

Ricordiamo che i DRG dei ricoveri per AIDS

"remunerativi" ed il decremento sensibile degenze ha significato, per le Az. USL, un delle U.O. di maluitie infettive.

di Bologna). 


\section{Nota xi}

Ricordiamo che il numero ridotto di pazienti che alla fine del 1999 era curato con due farmaci (circa il $15 \%$ della casistica dei circa 700 pazienti seguiti) nel corso del corrente anno si è sensibilmente ridimensionato: infatti ora quasi tutti i pazienti sono trattati con tre farmaci, adeguandosi così alle linee guida internazionali (8).

\section{Nota xii \\ Un calcolo effettuato nell'agosto 2000 in relazione alla spesa registrata nel I semestre, dimostra che l'U.O.M.I. ha già speso per farmaci 3.801 milioni pari al 48,3\% del bugdet fissato per questa voce per il corrente anno. Lo stesso calcolo, eseguito nel giugno '99, riportava una spesa pari a 3.687 milioni.}

\section{Nota xiii \\ In un'indagine effettuata nel 1999 abbiamo stabilito che su 287 ricoverati in reparto di degenza nel primo semestre, in 188 pazienti $(65.5 \%)$ si sono utilizzati antibioti- ci con una spesa complessiva di $£$. 125.818.218 (49,1\% della sposa totale per antibiotici nel 1999)} (13). stabilire sia il numero di side effects scatenati da queste nuove molecole, sia il reale risparmio economico ottenuto, per singolo caso trattato, nel medio-lungo periodo.

Diverso è il ragionamento quando si considera il possibile risparmio nella U.O.M.I. in relazione agli altri farmaci ed in particolare rispetto all'utilizzo degli antibiotici. Infatti antibiotici e antimicotici sono utilizzati in termini quasi assoluti nel reparto di degenza ${ }^{\text {xiii }}$. Ci sono fondati motivi per ritenere che, a causa dell' adozione di protocolli terapeutici standard, nelle principali patologie trattate dall'U.O.M.I. (polmoniti, meningiti, endocarditi e setticemie) difficilmente saranno raggiungibili obiettivi di riduzione di spesa nel breve periodo. Invece il costo di tali trattamenti potrebbe ridursi (sensibilmente) se si ottenesse, in un numero considerevole di casi, l'obiettivo strategico di una dimissione ospedaliera anticipata, con la prosecuzione della terapia a domicilio. La classe dei chinolonici, e in particolare la ciprofloxaeeina ci è sembrata utilizzabile con successo nelle polmoniti (13). L'intento è quello di anticipare la dimissione ospedaliera, trattando il paziente a domicilio con una molecola potente, disponibile per uso E.V. e per via orale. Numerosi altri studi indicano questa prospettiva come strategicamente interessante $(14,15)$.

Con la modalità di cura "schift terapy" (S.T.) associata alla dimissione anticipata non solo migliorerebbe la spesa farmaceutica ospedaliera, ma si ridurrebbe significativamente anche il costo complessivo delle degenze, con il ridimensionamento della durata media, nella prospettiva indicata anche dal Piano Sanitario Regionale (16). Si salvaguarderebbero così gli obiettivi di qualità e di produttivitàn ${ }^{x i v}$. Ricordiamo che, per praticare la S.T., è necessario che il paziente, dimesso anticipatamente, possa contare su una famiglia o su una struttura (RSA) che possa garantire una buona assistenza domiciliare. Inoltre, per ottenere il risultato strategico di ridurre la degenza ospedaliera mediante l'adozione di S.T., dovrebbe migliorare, a nostro avviso, la collaborazione tra medici di famiglia e medici ospedalieri.
In riferimento alla spesa per antinfettivi nell'ambito dell'intera Azienda USL, che ricordiamo consta di due presidi di medie dimensioni, (dotati di specializzazioni mediche e chirurgiche), di reparti di eccellenza e di diversi Day Hospital, una valutazione in merito alle possibilità di riduzione della spesa farmaceutica è di estrema difficoltàxv. Nell'attuale fase sarebbe interessante l'adozione, nei diversi reparti, di protocolli terapeutici che permetterebbero l'abolizione degli utilizzi irrazionali ed obsoleti, con l'obiettivo di innescare un circolo virtuoso. Adottando questa scelta strategica, non solo migliorerebbe l'approccio terapeutico per le patologie infettive, ma verosimilmente trarrebbe vantaggio anche questa voce del budget aziendale.

È auspicabile pertanto l'adozione in tutta l'Azienda di linee guida per le più importanti e frequenti patologie infettive. La costituzione di gruppi di lavoro ad hoc potrebbe essere una strada perseguibile, in particolare se i risultati delle riflessioni sviluppate, una volta divulgate nelle varie U.O., fossero discusse e si valutasse, nel tempo, il grado di adesione a quanto raccomandato. Per cultura ed esperienza specifica dovrebbero essere gli infettivologi a coordinare questi gruppi di lavoro.

Il processo di riqualificazione della sanità, di cui l'accreditamento è un fattore portante, potrebbe trarre vantaggio da momenti riflessivi comuni di tipo teorico-pratico nell' ambito dell'azienda. Così i professional, interessati al miglioramento complessivo, potrebbero confrontarsi anche sulla qualità del loro contributo, con un occhio attento ai conti e alla qualità del servizio pubblico fornito.

Attraverso il processo di riforma innescato sarà possibile coniugare la riqualificazione del ruolo medico e dei processi produttivi con il miglioramento dei conti della sanità pubblica, anche se crederci è molto difficile, dopo anni di delusioni e frustrazioni.

\author{
Nota xiv \\ In Emilia Romagna la Commissione Regionale per l'accreditamento delle U.O. Malattie Infettive (A.M.O.L.) in Linee Guida \\ divulgate nel 2000 ha fissato tra i requisiti minimi di attività i seguenti parametri: durata degenza massima $<12$ giorni; Indice di \\ rotazione: >20; Indice di occupazione posti letto: = >70\% per $\mathrm{i}$ Reparti di degenza e $100 \%$ per il D.H..
}

\title{
Nota Xv
}

In Italia nel 1999 la spesa generale per farmaci antinfettivi è stata di 1174 milioni di dollari pari al 12\% del mercato farmaceutico italiano; questa classe terapeutica è risultata la terza dopo i farmaci per il sistema cardio-vascolare (24\%) ed i farmaci per l'apparato gastrointestinale ed il metabolismo (15\%) (17). 


\section{BIBLIOGRAFIA}

1. Lega F.: Lineamenti di teoria per la strategia ed il cambiamento organizzativo nelle aziende sanitarie pubbliche: Pianificazione Vs. Apprendimento Organizzativo, SDA Bocconi. Articolo fornito al corso per Manager Aziendali, Maggio 2000. Milano.

2. Tramarin A.: Le basi metodologiche e le implicazioni cliniche dell'approccio economico alla farmacoterapia, EDIMES. PAVIA 1999.

3. Eandi M., Bondonio P.V., Dirindin M.: Farmacoeconomia e terapia antibiotica, A.B.E. Edizioni scientifiche, 1993 Milano.

4. Eisemberger J.M., Glick H., Koffer H.: PharmacoEconomics: Economics evaluation of pharmaceuticals, (in B. Strom Ed.) Pharmacoepidemiology. NewYork: Churchil Livingston, 1989.

5. Guidelines for Economic Evaluation of Pharmaceuticals Canada, $2^{\text {th }}$ Edition November 1997. Canadian Coordinating Office for Health Technology Assesment.

6. Muir Gray J.A.: Evidence - based health care, London: Churchill Livingstone, 1997.

7. Tolley K.: Health Promotion: how to measure cost-effectiveness, Health Education Authority, Hamilton House, 1994 London.

8. Carpenter C.C.J. et al.: Antiretrovirai therapy in Adults, Updated hecommendations of the International AIDS Society, USA Panel - JAMA 3 - V 283;381,390,2000.

9. Montaner J.S.G., Reiss P., Cooper D., Vella S. et al., for the INCAS Study Group: Long-term follow-up of patients treated with Nevirapine (Nvr) based combination therapy within the INCAS trial. 12 $2^{\text {th }}$ World Aids Conference, Geneva, 1998. Abstract 12,368.

10. Murphy R., Katlama C., Tohnson V. et al.: the Atlantic Study: A randomized, open-label trial comparing two protease inhibitors (P.I.), sparing antiretrovirai strategics versus a standard P.I., - containing regimen, 48 week data $39^{\text {th }}$ ICAAC, San Francisco, 1999. Abstract LB - 22.

11. Staszewski et al.: Efavirenz plus Zidovudine and Lamivudine, Efavirenz plus Indivinavir and Indivinavir plus Zidovudine and Lamivudine in the treatment of HIV-1 infection in Adults. The N. Eng. Jour. Med. 341, 65-74, 19

12. Siabr et al.: Combination therapy with Efavirenz, Nelfinavir and Nucleoside Reverse. Transcriptase Inhibitors in Children Infected with Human Immunodeficiency Virus Type 1. N,Engl. Jour, of Med. 341, 74-81, 1999.

13. Sabbatani S., Cesari R., Pipitone E., Martelli L.:Alcune valutazioni farmacoeconomiche relative all'antibioticoterapia in un reparto di malattie infettive, in stampa sul n. 4 anno 2000 di "Le Infezioni in Medicina.

14. Nathwani D.: Sequential switch therapy for lower respiratory tract infections. A European perspective, Chest 1998; 113: $211 \mathrm{~s}-218$ gs.

15. Paladino J.A.: Phannacoeconomic comparison of sequential IV/oral Ciprofloxacin Versus ceftazidime in the treatment of nosocomial pneumoniae,Canad. Jour. Hosp. Pharm. 48, N. 5, 276-283.

16. Piano Sanitario Regionale 1999-2001: Il Patto di solidarietà per la salute in Emilia Romagna. Il ruolo della rete, ospedaliera regionale, Proposte di linee guida, Bologna, 11 febbraio 2000,

17. Bollettino d'informazione sui farmaci, Il mercato farmaceutico mondiale nel 1999, anno Vll 2,45-46, 2000. 